\title{
Guest Editorial 2020 Custom Integrated Circuits Conference
}

\author{
Q. Jane Gu, Senior Member, IEEE, Mark S. Oude Alink, Senior Member, IEEE
}

T HIS Special Issue of the IEEE JOURnAL OF SOLIDSTATE CIRCUITS features expanded versions of key articles presented at the 2020 Custom Integrated Circuits Conference (CICC), one of IEEE's first conferences to go fully virtual due to the corona virus pandemic, from March 22 to March 25, 2020. Originally planned to be held at Hyatt Boston Harbor, Boston, MA, USA, growing concerns related to COVID-19 and the impact on the community's ability to travel to the conference lead the conference organization to make the tough decision in January 2020 to go for a fully virtual format. The dates and schedule of the conference remained unchanged.

Although going virtual, the CICC remains a vibrant forum for sharing the state-of-the-art techniques from worldrenowned experts in custom IC design and adjacent fields. Notably, CICC demonstrates its commitment to its mission to educate and disseminate information by making the Sunday tutorial track free for all registered conference attendees.

A total of 19 high-quality articles were selected for an extended version in this journal, allowing for a more indepth presentation of the concepts, ideas, and test results than was available in the CICC conference digest. Together, they provide for an excellent sampling of the broad range of topics in IC design, from analog/RF/mmWave to mixed-signal and digital designs, from elementary building blocks to complete SoCs, and from sensors and actuators to data and power converters to wireless and wireline communication.

The Guest Editors would like to thank the authors for contributing their articles and meeting the tight deadlines required for this Special Issue. They also thank many anonymous reviewers whose feedback and comments ensure the high quality of the journal, the present JSSC Editor-in-Chief P. Hanumolu for his guidance, and finally, the JSSC administrators for their assistance in publishing this issue.

We hope that this JSSC Special Section will provide a useful cross section of the state of the art in IC design at the circuit, system, and application levels. We would also like to encourage readers to attend the 2021 CICC, which will again be fully virtual, to be held on April 25-28, 2021.

Q. JANE GU, Guest Editor University of California, Davis

Davis, CA 95616 USA jgu@ucdavis.edu

\section{MARK Oude Alink, Guest Editor} University of Twente

Enschede, 7522NB Netherlands m.s.oudealink@utwente.nl

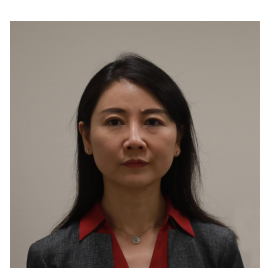

Qun Jane Gu (Senior Member, IEEE) received the $\mathrm{Ph}$.D. degree from the University of California at Los Angeles, Los Angeles, CA, USA, in 2007.

After a couple of years industry experience, she started her academia career in 2010 at the University of Florida, Gainesville, FL, USA. Since 2012, she has been with the University of California at Davis, Davis, CA, USA. Her research interests include high-efficiency, low-power interconnect, millimeterwave and sub-millimeter-wave integrated circuits, system-on-a-chip design techniques, and integrated terahertz circuits and systems for communication, radar, and imaging.

Dr. Gu was a recipient of the 2013 NSF CAREER Award, the 2015 COE Outstanding Junior Faculty Award, the 2017 and 2018 Qualcomm Faculty Award. Her group has won eight best paper awards, including the Best Student Paper Award of the 2015 IEEE Asia-Pacific Microwave Conference (APMC), the Best Student Paper the Second Place of the 2016 IEEE MTT-S International Microwave Symposium (IMS), and the Best Student Paper the Third Place of the 2017 IEEE IMS and 2020 IEEE IMS respectively.

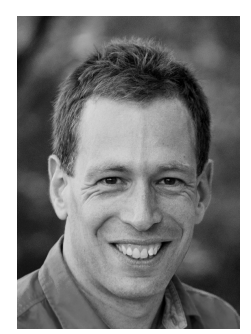

Mark S. Oude Alink (Senior Member, IEEE) received M.Sc. degrees in electrical engineering and computer science in 2008 (both cum laude) and a $\mathrm{Ph} . \mathrm{D}$. degree in 2013 (cum laude), from the University of Twente, Enschede, the Netherlands.

After several years in industry as system and RFIC design engineer, he returned to his alma mater as assistant professor within the IC-Design group. His research interests includes wireless communications, low-power analog, mixed-signal and RF circuits and systems.

Dr. Oude Alink was a recipient of the prestigious Else Kooi award in 2013 He has been serving on the Technical Program committee of the Custom Integrated Circuits Conference (CICC) since 2018. 